\title{
Sorafenib inhibits proliferation and invasion of human hepatocellular carcinoma cells via up- regulation of p53 and suppressing FoxM1
}

\author{
Ji-chao WEI ${ }^{1}$, Fan-di MENG ${ }^{1}$, Kai QU ${ }^{1}$, Zhi-xin WANG ${ }^{1}$, Qi-fei WU ${ }^{2}$, Ling-qiang ZHANG ${ }^{1}$, Qing PANG ${ }^{1}$, Chang LIU ${ }^{1,}$ * \\ ${ }^{1}$ Department of Hepatobiliary Surgery, ${ }^{2}$ Department of Thoracic Surgery, The First Affiliated Hospital of Xi'an Jiaotong University, Xi'an \\ 710061, China
}

\begin{abstract}
Aim: Forkhead box M1 (FoxM1) is a transcription factor that plays important roles in the pathogenesis and progression of human cancers, including hepatocellular carcinoma (HCC). The aim of this study was to examine the involvement of FoxM1 in the anti-cancer action of sorafenib, a multikinase inhibitor, in human HCC cells.

Methods: HCC cell lines HepG2 and HuH-7 were tested. Cell viability was examined using MTT assay and cell invasion was determined with Transwell migration assay. The relevant mRNA expression was determined with RT-PCR, and the proteins were detected using Western blotting and immunofluorescence assays. RNA interference was used to modify the expression of p53 and FoxM1. HuH-7 cell line xenograft mice were used for in vivo study, which were treated with sorafenib $(40 \mathrm{mg} / \mathrm{kg}$, po) daily for 3 weeks.

Results: Sorafenib (2-20 $\mu \mathrm{mol} / \mathrm{L})$ inhibited the proliferation of the cells in dose- and time-dependent manners with an $\mathrm{IC}_{50}$ value of nearly $6 \mu \mathrm{mol} / \mathrm{L}$ at $48 \mathrm{~h}$. Sorafenib ( $6 \mu \mathrm{mol} / \mathrm{L}$ ) markedly suppressed the cell invasion. Furthermore, sorafenib (2-6 $\mu \mathrm{mol} / \mathrm{L})$ dose-dependently decreased the expression of FoxM1, MMP-2, and Ki-67, and up-regulated that of p53 in the cells. Silencing p53 abolished the decrease of FoxM1 and increase of p53 in sorafenib-treated cells. Silencing FoxM1 significantly reduced the expression of MMP-2 and Ki-67, and enhanced the anti-proliferation action of sorafenib in the cells, whereas overexpression of FoxM1 increased the expression of MMP-2 and Ki-67, and abrogated the anti-proliferation action of sorafenib. In the xenograft mice, sorafenib administration decreased the tumor growth by $40 \%$, and markedly increased the expression of p53, and decreased the expression of FoxM1, MMP-2, and Ki-67 in tumor tissues.

Conclusion: Sorafenib inhibits HCC proliferation and invasion by inhibiting MMP-2 and Ki-67 expression due to up-regulation of P53 and suppressing FoxM1.
\end{abstract}

Keywords: hepatocellular carcinoma; sorafenib; p53; FoxM1; MMP-2; Ki-67; RNA interference; tumor xenograft model

Acta Pharmacologica Sinica (2015) 36: 241-251; doi: 10.1038/aps.2014.122; published online 5 Jan 2015

\section{Introduction}

Hepatocellular carcinoma (HCC) is the fifth most common carcinoma and the third leading cause of cancer-related death worldwide. During the past decades, the incidence of HCC has increased. At an early stage, surgical resection is the most effective treatment. Other treatment options include orthotopic liver transplantation (OLT) and radiofrequency ablation (RFA). Following tumor resection, the HCC recurrence rate can be as high as $50 \%$ at 2 years $^{[1]}$. Most HCC patients are at an advanced stage of disease at the time of diagnosis. In these cases, systemic pharmacotherapy is the primary treatment. However, the long-term effectiveness of most chemotherapeu-

\footnotetext{
* To whom correspondence should be addressed.

E-mail liuchangdoctor@163.com

Received 2014-08-05 Accepted 2014-10-30
}

tic drugs is often hampered by the development of acquired drug resistance ${ }^{[2]}$. Several medical centers have provided data showing that traditional systemic chemotherapy is poorly tolerated and yields low objective response rates $(<10 \%)$ without a proven survival benefit. Acquired chemo-resistance has significantly limited clinical applications of drug therapies and may contribute to the high recurrence rate. Therefore, it is necessary to investigate the molecular pathogenesis of HCC to develop novel targeted therapies against this fatal disease.

Recently, clinical investigations have shown that sorafenib, a multikinase inhibitor, may represent a breakthrough in the treatment of HCC, particularly unresectable $\mathrm{HCC}^{[3]}$. Sorafenib has been approved by the Food and Drug Administration (FDA), has been shown to be effective against several solid tumors and is a standard treatment for $\mathrm{HCC}^{[4]}$. Previous studies have reported that sorafenib blocks platelet-derived growth 
factor (PDGF), vascular endothelial growth factor (VEGF), Fms-like tyrosine kinase-3 (FLT3), c-Kit, and Raf signaling ${ }^{[5]}$ in both tumor cells and the surrounding endothelial cells ${ }^{[6]}$. The underlying mechanism is believed to involve competitive inhibition of ATP binding to the catalytic domains of the various kinases $^{[7]}$. Preclinical experiments have shown that sorafenib has strong anti-proliferative properties and decreases tumor invasion. Apoptosis induction is also believed to mediate the anti-cancer effects of sorafenib in HCC cells. A previous study demonstrated that the down-regulation of the anti-apoptotic protein myeloid cell leukemia 1 (Mcl-1) and inhibition of eukaryotic translation initiation factor $4 \mathrm{E}$ (eIF4E) phosphorylation contribute to sorafenib-induced apoptosis ${ }^{[8]}$. However, because triggering apoptosis by deleting Mcl-1 alone in solid tumor cells is often insufficient, the anti-cancer mechanism that underlies the effect of sorafenib has not yet been completely elucidated.

Forkhead box M1 (FoxM1), a member of the Forkhead box (Fox) family of transcription factors, has been reported to play important roles in the pathogenesis and progression of many human cancers, including $\mathrm{HCC}^{[9,10]}$. Suppression of FoxM1 expression can lead to mitotic spindle defects, chromosome disaggregation, mitotic catastrophe, and cell cycle arrest ${ }^{[11]}$. FoxM1 is a key regulator in the $G_{1} / S$ and $G_{2} / M$ transitions and $\mathrm{M}$ phase progression. FoxM1 has roles in cross-talk with multiple oncogenic signaling pathways ${ }^{[12]}$ and regulates the expression of several cell cycle regulators, including $\mathrm{p} 21^{\mathrm{Cip} 1}$, p27 ${ }^{\mathrm{Kip} 1}$, cyclin B, CDC25B, aurora B kinase, survivin, and pololike kinase-1 (PLK1) ${ }^{[13]}$. In addition, the phosphorylation of FoxM1 via the Raf/MEK/MAPK pathway stimulates FoxM1 nuclear translocation and thereby its transcriptional activity during $\mathrm{G}_{2} / \mathrm{M}^{[14]}$. Evidence has suggested that FoxM1 is a reliable target for arresting cancer growth and progression. In carcinogenic mouse models, the depletion of FoxM1 can significantly reduce cancer cell proliferation and tumor growth in many solid tumors. By contrast, a marked increase in the proliferation, number and size of tumors was observed when mice with the FoxM1 transgene were subjected to tumor induction by carcinogens ${ }^{[15]}$. FoxM1 can promote tumor angiogenesis by activating VEGF expression through direct binding to Forkhead binding elements (FHRE) of the VEGF promoter ${ }^{[16]}$. Down-regulation of FoxM1 has been shown to inhibit invasion and angiogenesis by reducing MMP-2 and MMP-9 expression $^{[17]}$. Previous studies have reported that senescence was observed in embryonic fibroblasts derived from FoxM1 knockout mice and that increased levels of FoxM1 counteracted $\mathrm{H}_{2} \mathrm{O}_{2}$-induced senescence ${ }^{[18]}$. Recent studies have revealed that FoxM1 participates in determining anti-cancer drug sensitivity and may promote the development of acquired drug resistance if aberrantly activated or expressed ${ }^{[19-21]}$.

In this study, we found that sorafenib kills HCC cells in vitro and in vivo by suppressing FoxM1 through the up-regulation of p53. Our results shed light on the anti-cancer mechanism of sorafenib and provide a framework within which FoxM1 can be manipulated to improve the efficacy of targeted therapy.

\section{Materials and methods}

\section{Cell culture and drugs}

The human HCC cell lines HepG2 and HuH-7 were purchased from Cell Bank of Shanghai Institutes for Biological Sciences, Chinese Academy of Sciences (Shanghai,China) and maintained in Dulbecco's modified Eagle's medium (DMEM) supplemented with $10 \%$ fetal bovine serum (FBS) in a humidified atmosphere of $95 \%$ air and $5 \% \mathrm{CO}_{2}$ at $37^{\circ} \mathrm{C}$. Sorafenib was obtained from Bayer Pharmaceuticals Corporation. Sorafenib was dissolved in DMSO at a concentration of $100 \mu \mathrm{mol} / \mathrm{L}$ for storage and was formulated at various concentrations (from 0 to $20 \mu \mathrm{mol} / \mathrm{L}$ ) for cell culture and in vivo studies. Cells were incubated with a sub-IC $\mathrm{IC}_{50}$ concentration of sorafenib. The $\mathrm{IC}_{50}$ values of each drug had been determined in previous experiments. All of the dosing solutions were prepared on the day of use with endotoxin-free distilled water and were vortexed immediately.

\section{Cell viability assay}

The HepG2 and HuH-7 cells were seeded at a density of 3000 cells per well in 96-well microtiter culture plates and incubated in DMEM with $10 \%$ FBS overnight. In the following day, the cells were treated with increasing concentrations of sorafenib. The controls received DMSO at a concentration equal to that of the sorafenib-treated cells. The effect of sorafenib pretreatment on cell viability was examined by the MTT assay.

\section{Western blot analysis}

Various concentrations of sorafenib were added to the cells that were incubated in six-well plates, and then the cells were harvested after $0,12,24$, or $48 \mathrm{~h}$ of treatment. The cells were washed twice in PBS, and lysates were prepared by suspending the cells with RIPA lysis buffer including protease and phosphatase inhibitors. The concentration of total protein was calculated using the Bio-Rad Protein Assay (Bio-Rad, Hercules, CA, USA). Equal volumes of protein samples were separated by $10 \%$ SDS-PAGE gel electrophoresis, followed by transfer to polyvinylidene difluoride membranes. Membranes were blocked in $5 \% \mathrm{BSA}$ and incubated at $4{ }^{\circ} \mathrm{C}$ overnight with the following polyclonal antibodies: rabbit anti-FoxM1 (1:400; Santa Cruz Biotechnology), rabbit anti-mmp2 (1:200; Santa Cruz Biotechnology), rabbit anti-ki-67 (1:400; Santa Cruz Biotechnology), and mouse anti- $\beta$-actin (1:200; Santa Cruz Biotechnology). Appropriate horseradish peroxidase-conjugated secondary antibodies were applied. Signals were visualized using an enhanced chemiluminescence detection system (BioRad). The quantitation of protein expression was performed using Imagelab software (Bio-Rad).

\section{Real-time PCR}

Total RNA from cells was isolated by TRIzol (Invitrogen) according to the manufacturer's protocol, and cDNA was synthesized using reverse transcriptase (Bio-Rad). Equal amounts of cDNA were used for all of the PCR assays (Promega). The forward and reverse primers used were 
as follows: p53-F: 5'-ATGAGCCGCCTGAGGTTGG-3', p53-R: 3'-CAGCCTGGGCATCCTTGAGT-5'; FoxM1F: 5' -TGCAGCTAGGGATGTGAATCTTC-3', FoxM1R: 3'-GGAGCCCAGTCCATCAGAACT-5'; MMP-2-F : 5'-CCAACTACAACTTCTTCCCTCGC-3', MMP-2-R: 5'-AGCAAAGGCATCATCCACTGTC-3'; and Ki-67-F: 5'-GGGAAAGTAGGTGTGAAAGAAGAG-3', Ki-67-R: 3'-ATCTGCTTTGGAGACTCCTTA-5'. Each RT-PCR assay was performed 6 times. The results are shown as the mean \pm SD.

\section{Cell invasion assay}

Cell invasion assays were performed using a Matrigel-coated transwell invasion chamber (BD Bioscience) according to the manufacturer's protocol. Briefly, the cells were pretreated with sorafenib for $48 \mathrm{~h}$ and then were trypsinized and resuspended in DMEM containing $0.1 \%$ bovine serum albumin. Next, the cells were added to the upper chambers at a concentration of $2.5 \times 10^{4}$ cells per well. DMEM containing $5 \%$ FBS was added to the lower chambers as a chemoattractant. After $24 \mathrm{~h}$, the invaded cells on the membrane's undersurface were stained with Giemsa's solution and counted in 5 fields per well. All of the experiments were conducted in triplicate and repeated twice.

\section{Immunofluorescence}

After appropriate treatment in six-well plates, the cells were fixed with ice-cold methanol for $10 \mathrm{~min}$ and permeabilized with $0.5 \%$ Triton X-100. Next, the samples were stained with rabbit monoclonal anti-FoxM1 antibody (Santa Cruz Biotechnology) overnight at $4^{\circ} \mathrm{C}$ and washed with PBS. For the immunofluorescence assay, the samples were incubated with FITC (green)-conjugated secondary antibodies (1:1000; Pierce, Rockford, IL, USA) and then observed with a laser scanning microscope (Olympus, Tokyo, Japan) using a magnification of $200 \times$. Nuclei were counterstained with DAPI. At least 6 randomly selected fields were examined in each of three separate experiments.

\section{RNA interference}

HCC cells were transfected with FoxM1 shRNA (GenePharma, Shanghai, China) and FoxM1 cDNA (GenePharma). To determine the role of FoxM1 in sorafenib treatment, we established two stable cell lines, HuH-7 LV-FoxM1 and HuH-7 LVshFoxM1, after lentivirus transduction. After 3 hours of incubation, cells were seed at a desired density in fresh medium and grown for the purpose of the experiment. HepG2 and HuH-7 cells $\left(2 \times 10^{6}\right.$ cells $\left./ \mathrm{mL}\right)$ were infected with lentivirus at an MOI of 10, respectively; at the same time, an empty carrier group and a blank control group were set up. Cells were seeded in $10-\mathrm{cm}$ plates and grown in medium supplemented with $1 \mathrm{mg} / \mathrm{mL}$ of G418 for $14 \mathrm{~d}$. The transfection liquid was discarded, and the medium was changed. Cells were then seeded in 96 -well plates at a low density ( 0.3 cell per well) to generate single-cell-derived colonies. The percentage of infected cells in each stable transformed cell line was calculated based on examination under an inverted fluorescence microscope (TE2000U; Nikon, Japan). A single-cell clone was transferred into a 24-orifice plate and allowed to expand, and the resulting cells were screened by G418 $(500 \mu \mathrm{g} / \mathrm{mL})$ for two months. Stable transfection of the clone cells could be obtained about two months later and were demonstrated by flow cytometry (Bio-Rad, CA, USA). Predesigned small interfering RNAs (siRNAs) that targeted p53 and Raf with scrambled sequences were purchased from GenePharma. Transfection was performed using the commercial protocols outlined by GenePharma. The growth of transfected cells with and without treatment was measured using the MTT assay.

\section{Tumor xenograft experiment}

The protocol for the in vivo studies was approved by the Institutional Animal Care and Use Committee of the College of Medicine, Xi'an Jiao tong University. Nude mice (6-8 weeks old) were purchased from the Laboratory of Animal Breeding and Research Center, Xi'an, China. The mice were inoculated subcutaneously (sc) at the right axilla with $1 \times 10^{6} \mathrm{HuH}-7$ cells in serum-free medium containing 50\% Matrigel (BD Biosciences; final volume, $0.1 \mathrm{~mL})$. The tumor volume was calculated using the following formula: volume $\left(\mathrm{mm}^{3}\right)=\mathrm{w}^{2} \times 1 \times 0.5$, where $w$ and $l$ refer to the smaller and larger dimensions collected at each measurement, respectively. The mice were then treated with orally administered sorafenib $(n=3,40 \mathrm{mg} / \mathrm{kg}$ per day) or a mock control $(n=3)$ for 3 weeks. The tumor volume and body weight were recorded every $5 \mathrm{~d}$.

\section{Immunohistochemistry (IHC)}

The samples were fixed in $10 \%$ formalin, embedded in paraffin, and sectioned ( $5 \mu \mathrm{m})$ for immunohistochemistry. The tumor sections were incubated $\left(65^{\circ} \mathrm{C}, 1 \mathrm{~h}\right)$, deparaffinized, steamed $\left(100^{\circ} \mathrm{C}, 120\right.$ atmospheres, $\left.40 \mathrm{~min}\right)$ for antigen retrieval, treated with $3 \% \mathrm{H}_{2} \mathrm{O}_{2}$ in methanol for $10 \mathrm{~min}$, blocked with rabbit monoclonal anti-FoxM1 antibody serum (diluted 1:20 in antibody diluent buffer; DAKO) and incubated with the primary antibodies (overnight, room temperature). Staining was performed using the Envision Plus HRP system from DAKO (Carpinteria, CA, USA) according to the manufacturer's protocol and counterstained with hematoxylin. The number of positive cells in the tumors was counted manually under a light microscope. Four fields per section were analyzed, excluding peripheral connective tissue.

\section{Statistical analysis}

Experiments were conducted in triplicate. Quantitative data are expressed as the mean $\pm S D$. Comparisons were calculated using Student's t-test. $P$-values were considered significant at $<0.05$.

\section{Results}

Cell proliferation and invasion are inhibited by sorafenib

To investigate the effects of sorafenib on the HCC cell lines, we treated HepG2 and HuH-7 cells with several doses (0-20 $\mu \mathrm{mol} / \mathrm{L})$ of sorafenib at different time points (0-48 h). The $\mathrm{IC}_{50}$ concentration was approximately $6 \mu \mathrm{mol} / \mathrm{L}$ (Figure 
1A). The cell viability of HepG2 and HuH-7 cells was significantly inhibited at $48 \mathrm{~h}$ (Figure 1B). Both cell lines were exposed to sorafenib at $6 \mu \mathrm{mol} / \mathrm{L}$. After $24 \mathrm{~h}$, the invaded cells on the membrane's undersurface were stained. A marked decrease in cell invasion was observed (Figure 1C and 1D). These data suggest that sorafenib can significantly inhibit cell growth and invasion in HCC cell lines. Previous studies have demonstrated that down-regulating the expression of FoxM1 can inhibit proliferation in various cells. We chose to further investigate the functional role of FoxM1 following sorafenib treatment in HCC cell lines.

\section{Sorafenib down-regulates FoxM1 levels}

To investigate the effect of sorafenib on FoxM1 expression, we examined the relative mRNA levels of FoxM1 in these two cell lines using real-time RT-PCR, and the expression of FoxM1, which was determined using Western blot analysis, was used to confirm the results. The FoxM1 mRNA levels and expression of FoxM1 protein were markedly reduced by sorafenib compared with control cells in a time- (Figure 2A and 2B) and dose-dependent (Figure 3A and 3B) manner. These data indicate that sorafenib partly inhibits HCC cell growth through the down-regulation of FoxM1.

\section{p53 plays a role in the effect of sorafenib on FoxM1}

To reveal the effects of down-regulating FoxM1 by sorafenib treatment, we tested other factors associated with cell proliferation and invasion. The mRNA levels of Ki-67 and MMP-2,
A

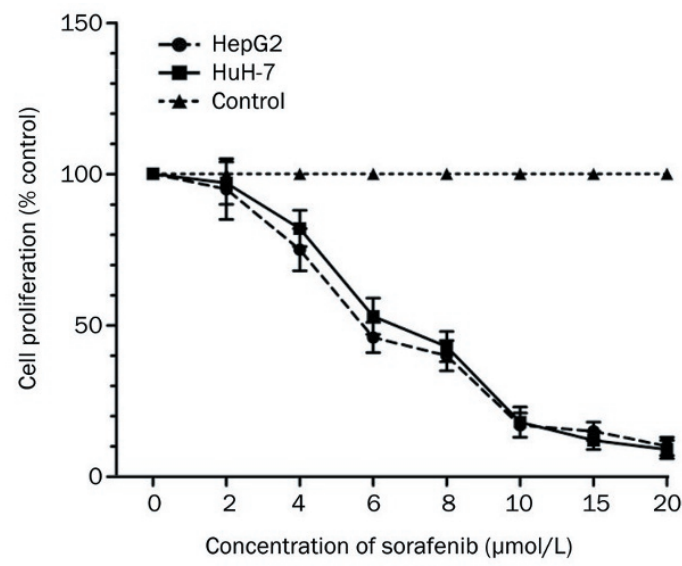

C
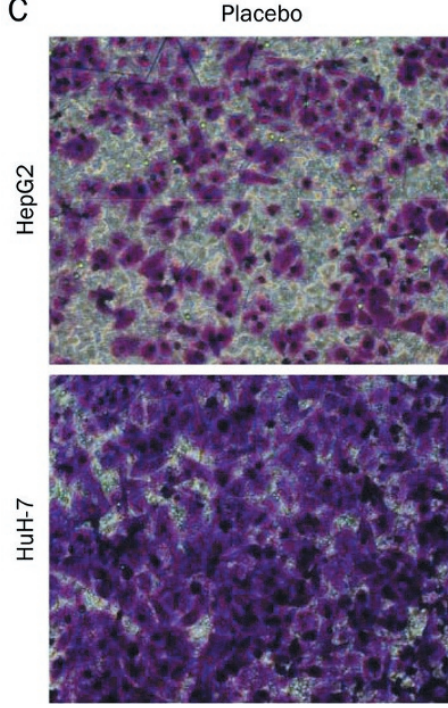

Sorafenib
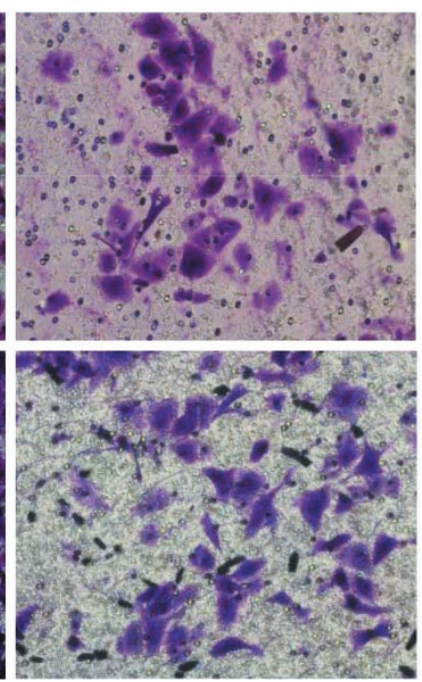

D
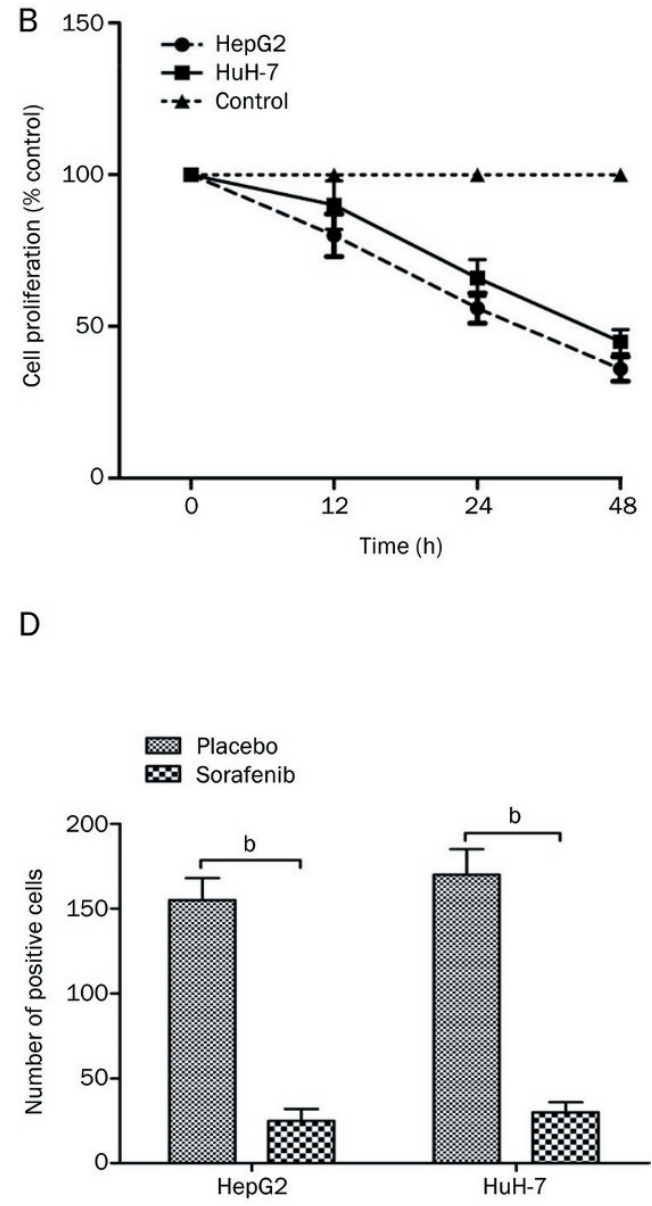

Figure 1. Sorafenib significantly inhibits the proliferation and invasion of HepG2 and HuH-7. The cell lines HepG2 and HuH-7 were exposed to sorafenib at increasing concentrations at $48 \mathrm{~h}$. The $\mathrm{IC}_{50}$ concentration was approximately $6 \mu \mathrm{mol} / \mathrm{L}$ (A). Cell proliferation was mostly inhibited at $48 \mathrm{~h}$ when both cell lines were exposed to sorafenib at $6 \mu \mathrm{mol} / \mathrm{L}(\mathrm{B})$. To eliminate the influence of cell death on the experiment, we included the control group without sorafenib treatment. The cell viability of the control group was set at $100 \%$ and was compared with that of other groups. The working concentration of sorafenib is $6 \mu \mathrm{mol} / \mathrm{L}$. The effect of sorafenib on cell invasion was tested by the Transwell assay (C). The number of positive cells is presented in (D). To each well of 24-well microtiter culture plates was added $500 \mu \mathrm{L}$ of medium that was used for cell invasion assay, and the volume of the $96-$ well microtiter culture plates used for the MTT assay was $100 \mu \mathrm{L}$ per well. The experiment shown is representative of at least three separate experiments. Statistical significance was defined as ${ }^{\mathrm{b}} P<0.05$. 
A

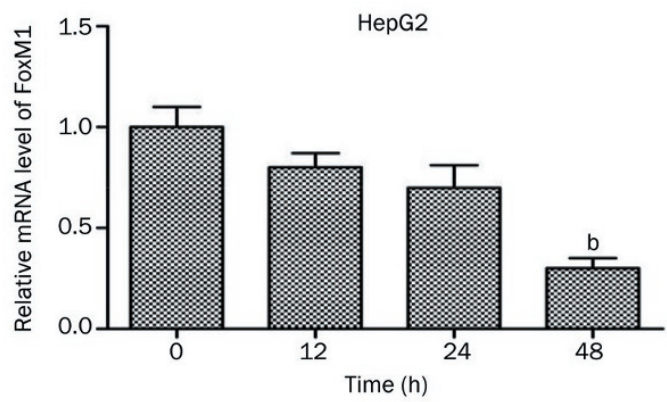

B

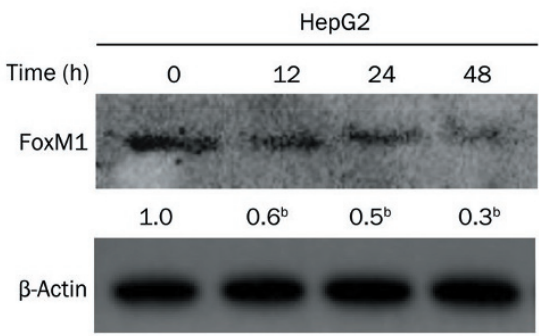

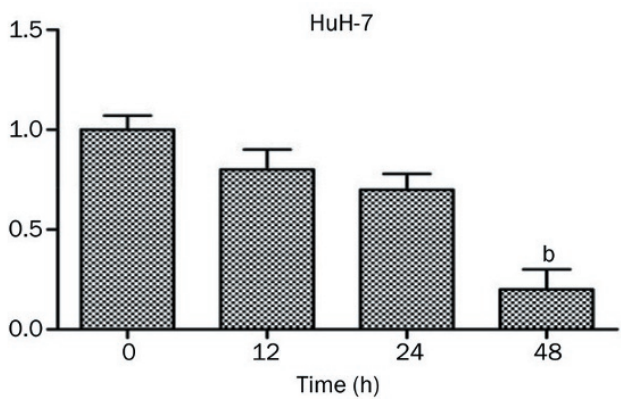

HuH-7

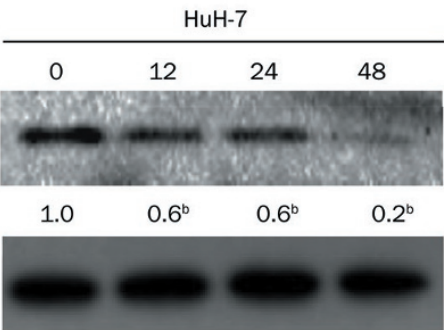

C
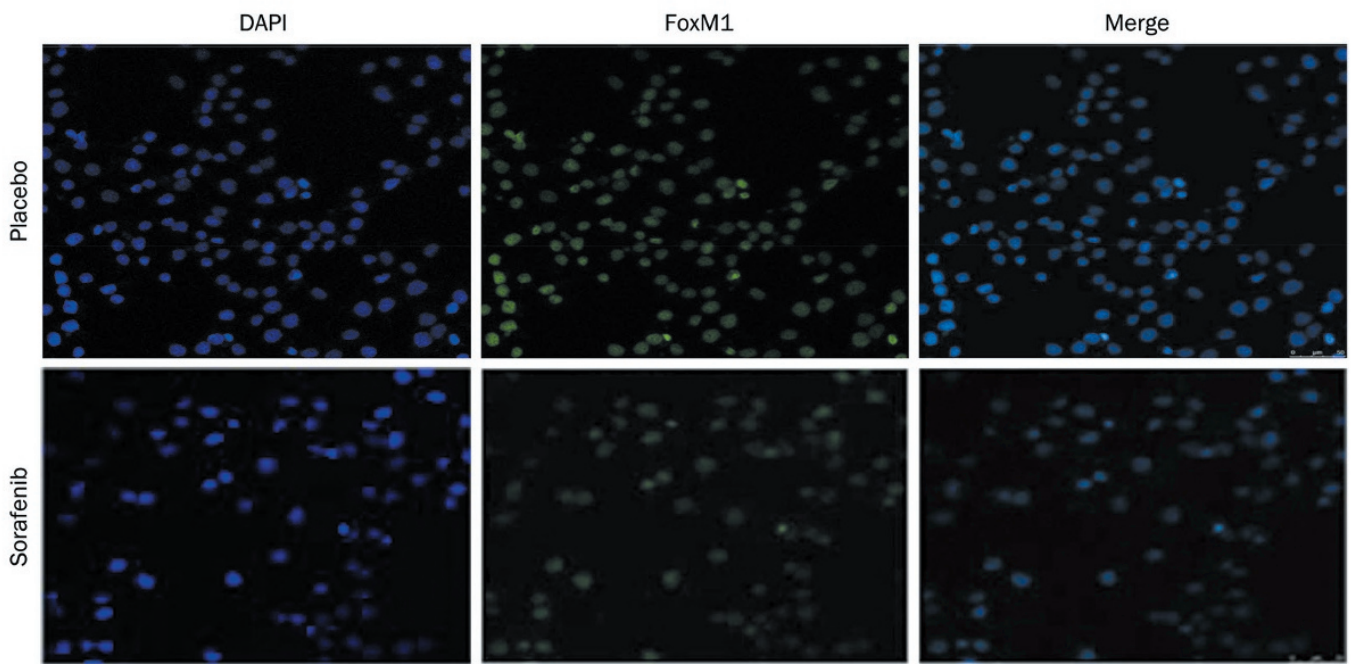

Figure 2. FoxM1 expression is inhibited by sorafenib in a time-dependent manner. FoxM1 mRNA levels in sorafenib-treated cells were evaluated by real-time PCR. Significant changes were observed in the FoxM1 mRNA levels after sorafenib treatment $(6 \mu \mathrm{mol} / \mathrm{L})$ in hepatoma cells at $48 \mathrm{~h}$ (A). The reduced FoxM1 expression following sorafenib treatment was observed by Western blotting and was time dependent (B). Equal sample loading was verified by stripping the blots and re-probing with anti $\beta$-actin antibody. The band density of each protein was normalized with $\beta$-actin and expressed as the mean \pm SD compared with the controls from 3 independent experiments. HuH-7 cells were treated with $6 \mu$ mol/L sorafenib for $48 \mathrm{~h}$. Immunofluorescence was conducted as described in the Materials and Methods for FoxM1 (green). Representative pictures are shown (C) with a magnification of $200 \times$. The experiment shown is representative of at least three separate experiments. At least six randomly selected fields were examined in each of three separate experiments. Statistical significance was defined as ${ }^{\mathrm{b}} P<0.05$.

which have been reported to promote cell survival, were decreased. These results were supported by Western blotting. However, p53 was elevated as determined by both real-time PCR and Western blotting (Figure 3A and 3B). These results suggest that the influences of sorafenib on HCC cell progression are partly mediated through the down-regulation of FoxM1, which can affect the expression of many other factors. To further explore the role of p53 in sorafenib-induced inhibition of FoxM1, we applied p53-specific siRNA to examine the influence of sorafenib. Silencing p53 promoted FoxM1 expression (Figure 4A) in the two HCC cell lines. However, no obvious differences were observed in p53 and FoxM1 expression with combined siRNA and sorafenib treatment (Figure 4B). The effect of sorafenib on FoxM1 appears to occur in a p53-dependent manner.

Sorafenib decreases HCC cell invasion and proliferation by inhibiting MMP-2 and Ki-67 expression through FoxM1 downregulation

The above results indicated that sorafenib inhibits the expression of FoxM1. As shown in Figure 3A, sorafenib apparently inhibits both the invasion and proliferation of HCC cells. The 

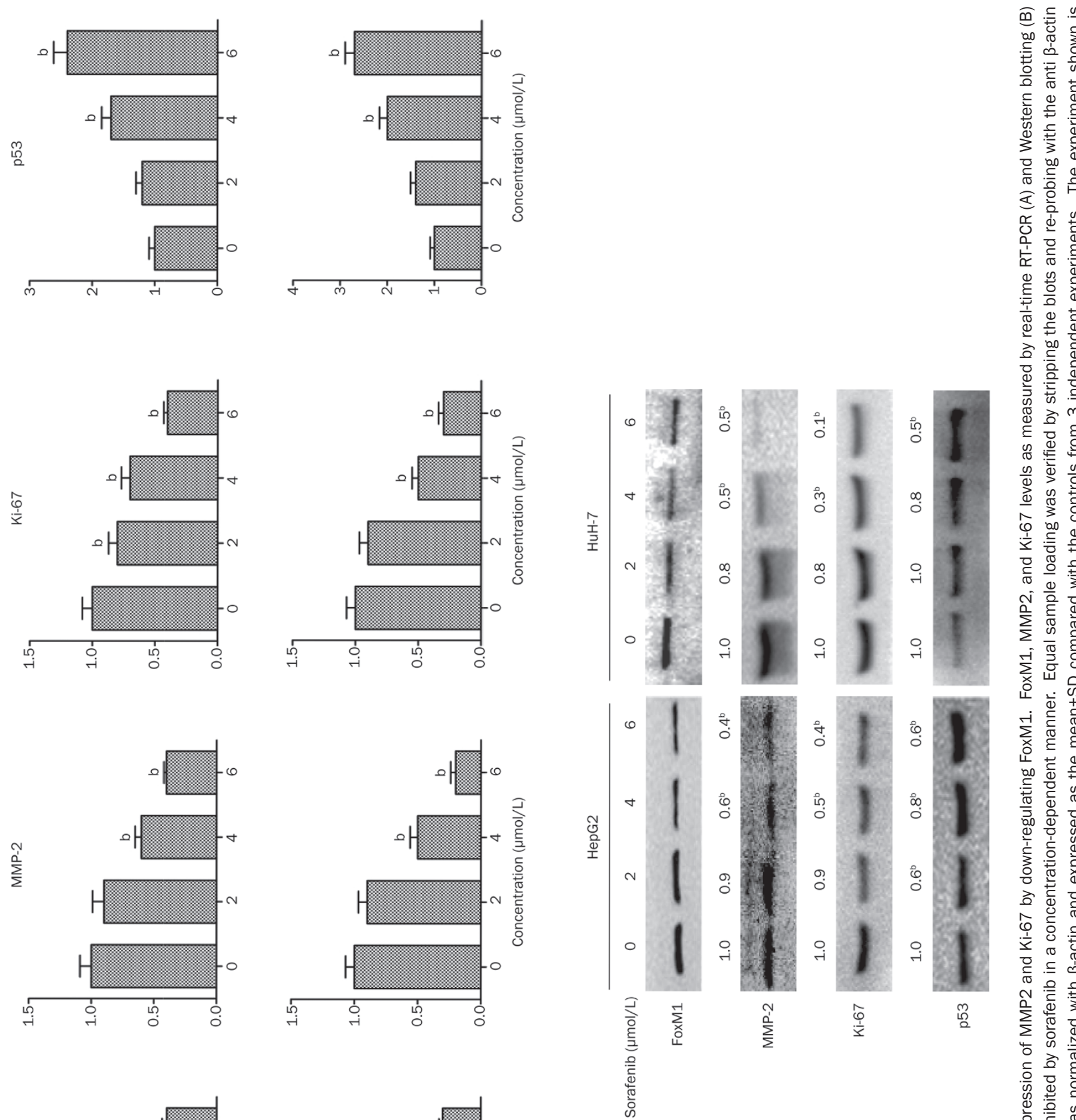

एँं

之ं

Oे

a)

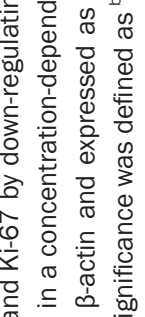

के

昌
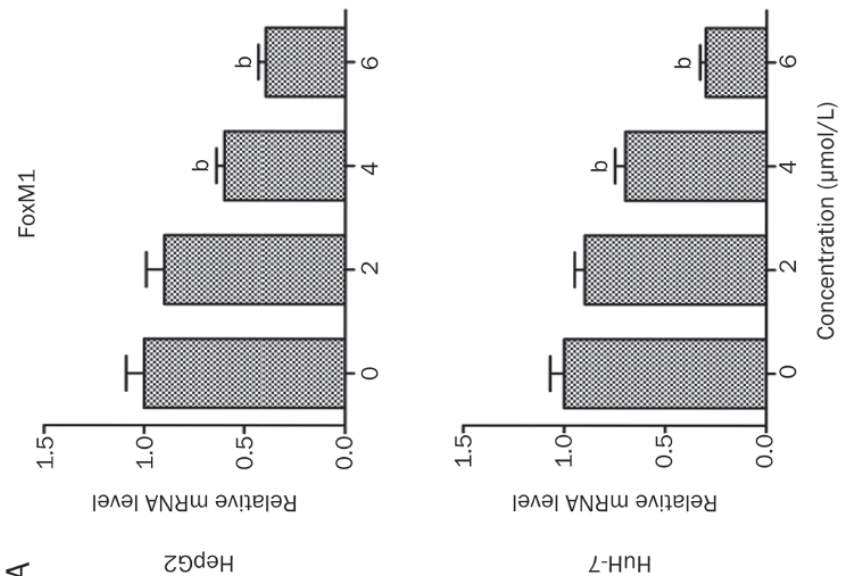

$\sum_{\sum}^{N}$

魰

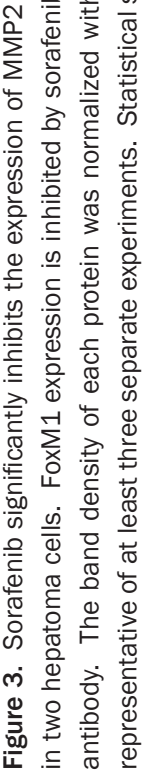


A

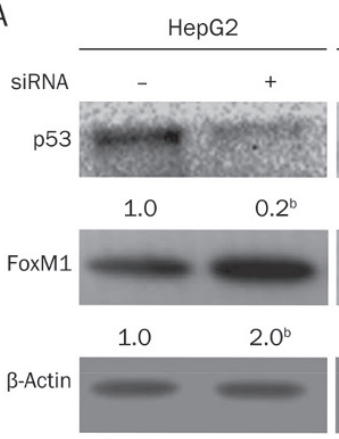

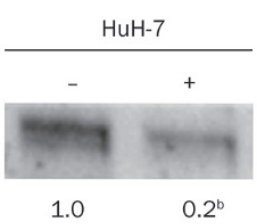

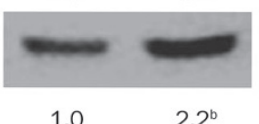

B

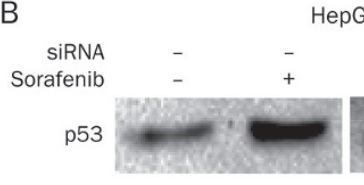

1.0

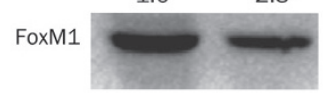

B-Actin
HepG2

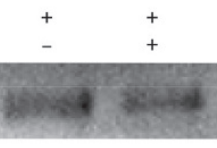

$1.0 \quad 1.1$

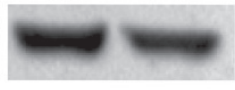

1.0

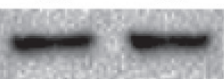

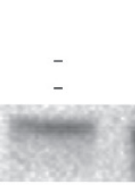

1.0

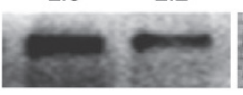

$1.0 \quad 0.5^{\mathrm{b}}$

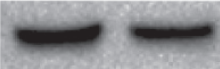

HuH-7

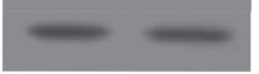

Figure 4. Regulation of p53 expression by siRNA, followed by sorafenib treatment. p53 down-regulation by siRNA can enhance FoxM1 expression in hepatoma cells (A). Down-regulation of p53 by siRNA, followed by sorafenib treatment as measured by Western blotting. p53 and FoxM1 expression was determined $(B)$. Equal sample loading was verified by stripping the blots and re-probing with the anti $\beta$-actin antibody. The band density of each protein was normalized with $\beta$-actin and expressed as the mean \pm SD compared with the controls from 3 independent experiments. The experiment shown is representative of at least three separate experiments. Statistical significance was defined as ${ }^{\mathrm{b}} P<0.05$.

expression of MMP-2 and Ki-67 was reduced as measured by Western blotting (Figure 3B). We asked whether FoxM1 could influence the expression of MMP2 and Ki-67. MMP-2 and $\mathrm{Ki}-67$ expression was significantly reduced in cells transfected with FoxM1 shRNA (Figure 5A). However, this trend was reversed after FoxM1 cDNA transfection (Figure 5B). We concluded that down-regulation of FoxM1 in HCC cells treated with sorafenib also leads to decreases in MMP-2 and Ki-67 levels.

Down-regulation of FoxM1 expression by shRNA can sensitize HCC cells to sorafenib

To determine the functional relevance of sorafenib-mediated alterations of FoxM1 expression in HCC cells, the effect of FoxM1 shRNA on cell viability was examined in HepG2 and HuH-7 cell lines using the MTT assay. Sorafenib treatment or shRNA transfection decreased the viability of HCC cells.
Interestingly, FoxM1 knockdown by shRNA in HepG2 cells led to a $>70 \%$ reduction in cell viability when used in combination with sorafenib treatment (Figure 6A). This effect was also observed in HuH-7 cells (Figure 6B). Collectively, these results indicate that targeting FoxM1 provides a method of sensitizing HCC cells to sorafenib treatment.

\section{Overexpression of FoxM1 by cDNA transfection enhances sorafenib resistance}

To study further the function of FoxM1 in HCC therapy, we transfected FoxM1 cDNA into HepG2 and HuH-7 cells which had been treated with sorafenib. FoxM1 expression was upregulated, resulting in a significant increase in cell growth and a decrease in sorafenib- induced cell inhibition. After $48 \mathrm{~h}$ of sorafenib treatment alone, the viability of the two cell lines was inhibited by approximately $50 \%$, whereas the proliferation of FoxM1 cDNA-transfected cells did not show any sig-
A

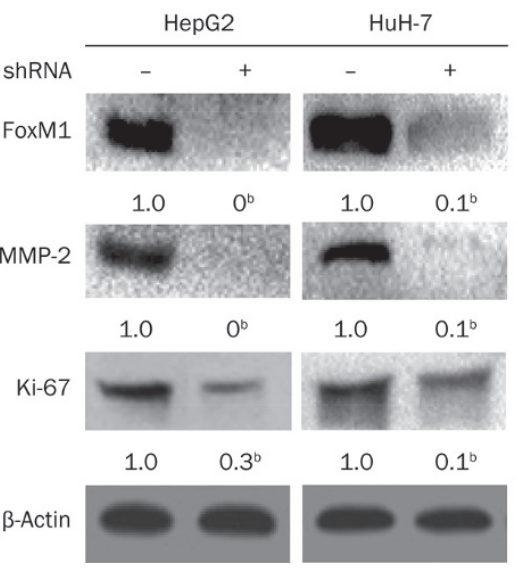

B

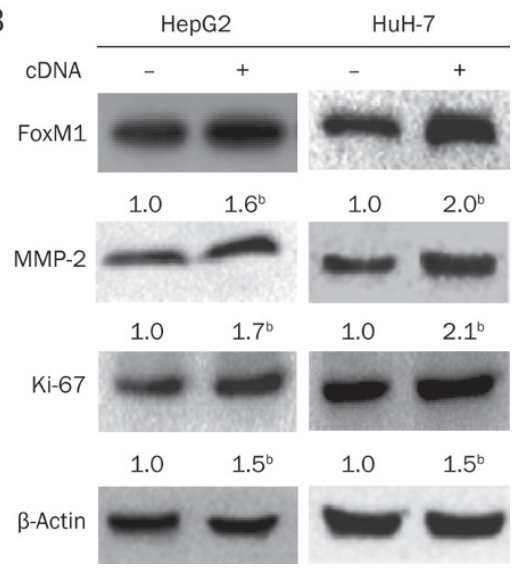

Figure 5. Effect of FoxM1 on cell invasion and proliferation factors. MMP-2 and Ki-67 expression as measured by Western blotting in two hepatoma cells transfected with shRNA or cDNA. MMP-2 and Ki-67 expression was significantly inhibited when the two cell lines were transfected with FoxM1 shRNA (A). MMP-2 and Ki-67 expression was enhanced when transfected with FoxM1 cDNA (B). No notable differences were observed in p53 expression. The band density of each protein was normalized with $\beta$-actin and expressed as the mean \pm SD compared with the controls from 3 independent experiments. The experiment shown is representative of at least three separate experiments. Statistical significance was defined as ${ }^{\mathrm{b}} P<0.05$. 
A

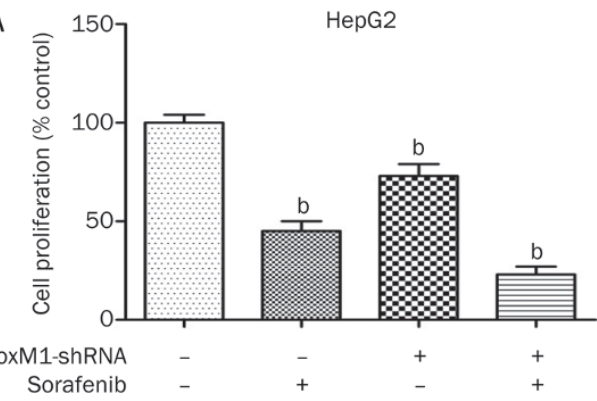

C

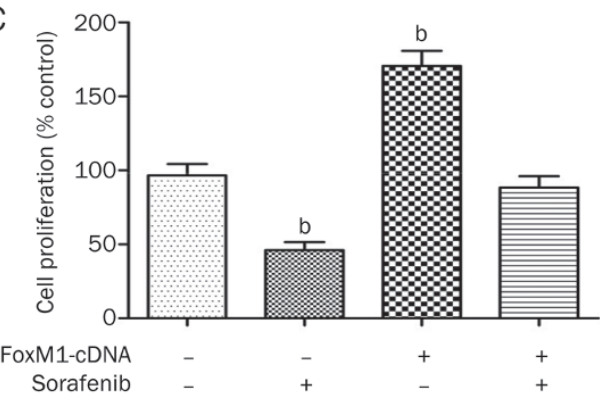

B

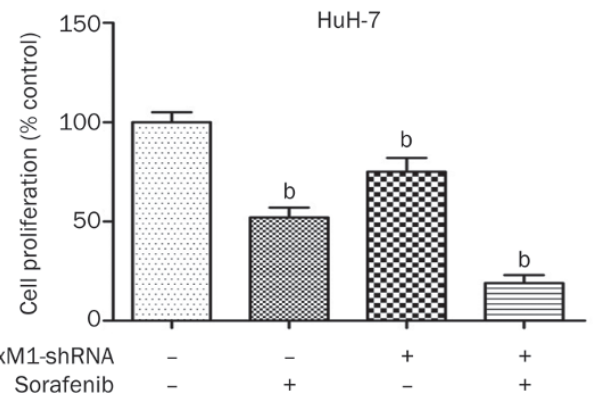

D

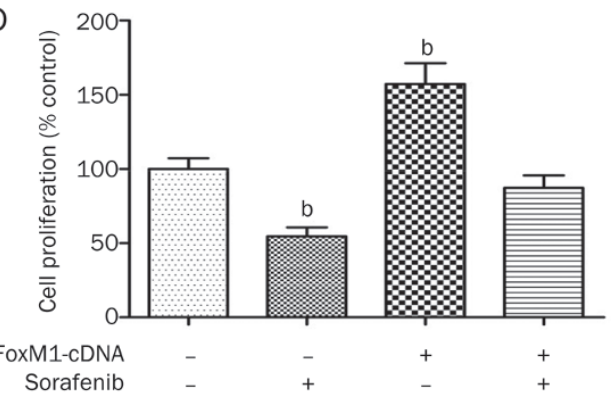

Figure 6. Regulation of FoxM1 expression by shRNA or CDNA, followed by sorafenib treatment. The growth viability of HCC cells was decreased by sorafenib treatment and shRNA transfection. Surprisingly, the proliferation of the two cell lines was inhibited by as much as $70 \%$ when sorafenib treatment was combined with shRNA transfection. Significant differences were observed compared with the no-treatment group. Down-regulation of FoxM1 expression by shRNA promoted sorafenib-induced cell growth inhibition in hepatoma cells (A and B). No obvious differences were observed when sorafenib treatment was combined with cDNA transfection. Overexpression of FoxM1 by FoxM1 cDNA transfection abrogated the sorafenibinduced cell growth inhibition in hepatoma cells ( $C$ and $D)$. The experiment shown is representative of at least three separate experiments. Statistical significance was defined as ${ }^{\mathrm{b}} \mathrm{P}<0.05$.

nificant decrease with sorafenib incorporation (Figure 6C and 6D). These data suggest that FoxM1 plays an important role in the resistance to sorafenib in HCC cell lines.

\section{Sorafenib down-regulates FoxM1 in vivo}

Sorafenib significantly inhibited tumor growth by $40 \%$ (Figure 7B). FoxM1 down-regulation also reduced tumor size (Figure 7A). However, the reduction was more significant in the HuH-7-FoxM1-shRNA stable cell group used in the xenograft model (Figure 7C). This reduction was not significant when FoxM1 expression was up-regulated in combination with sorafenib treatment (Figure 7D). To explore the underlying mechanism, we tested FoxM1 by IHC (Figure $7 \mathrm{E})$ and found that FoxM1 was clearly reduced. We further investigated the expression of effectors of FoxM1, such as MMP-2 and Ki-67, and found that their levels were decreased. However, p53 expression was elevated. These data suggest that FoxM1 is one of the most important molecular targets by which sorafenib potentiates its antitumor activity.

\section{Discussion}

The forkhead box family, which consists of more than 50 mammalian proteins including FoxM1, is important for a wide spectrum of biological processes, including metabolism, proliferation, apoptosis, migration, invasion, and longevity $^{[19]}$. FoxM1 is an oncogenic transcription factor that is overexpressed in various aggressive human cancers, including $\mathrm{HCC}^{[10]}$. Several studies have suggested that FoxM1 is a critical regulator of cell cycle progression. Furthermore, the activation of FoxM1 up-regulates its downstream target genes, resulting in the activation of cell proliferation, migration, invasion, and angiogenic processes, and also plays important roles in organogenesis, senescence and tumorigenesis ${ }^{[13,22]}$. In vitro, the deletion of FoxM1 leads to cell cycle arrest and is associated with mitotic spindle damage. In vivo, the loss of FoxM1 results in lethal embryonic effects due to the failure to enter mitosis. Interestingly, recent studies have indicated that FoxM1 is a valid target in drug-resistant tumors and that down-regulation of FoxM1 can sensitize tumor cells to drug treatment ${ }^{[20,21]}$. The addition of a FoxM1 inhibitor to chemotherapeutic regimens could lead to the use of lower effective doses and reduced side effects for patients. These studies indicate that FoxM1 is an attractive target for HCC therapy. Furthermore, recent studies have shown that FoxM1 overexpression is correlated with multiple malignant characteristics and indicates a poor prognosis for HCC patients after curative liver resection ${ }^{[23]}$. In addition, some pathological factors that contribute to the activation of FoxM1, such as chronic infection with hepatitis B virus, are also risk factors for HCC recurrence $^{[24]}$.

Several studies have investigated FoxM1 inhibitors. The antibiotics siomycin $\mathrm{A}$ and thiostrepton have been shown to 
A

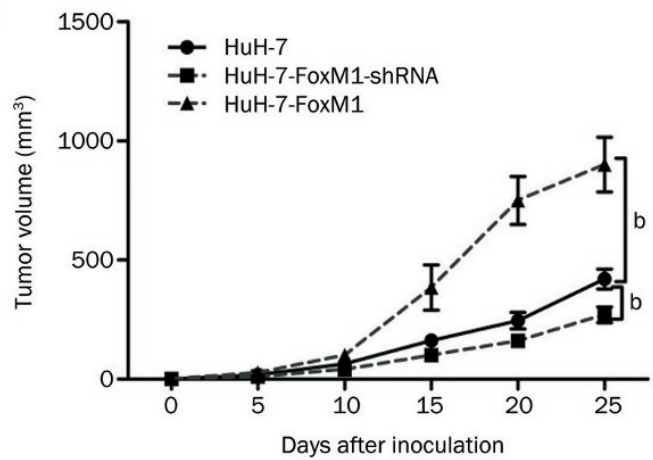

C

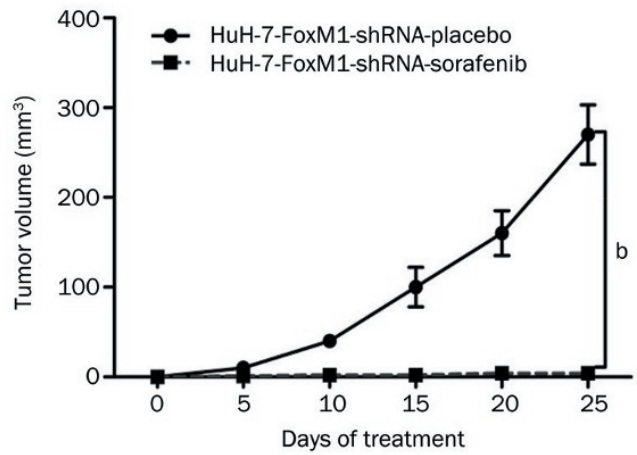

B

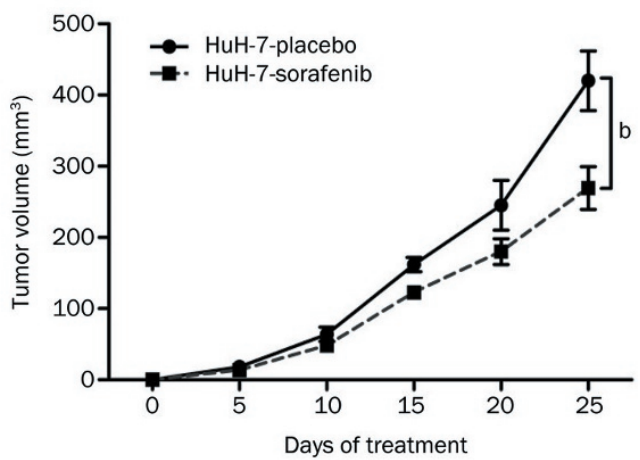

D

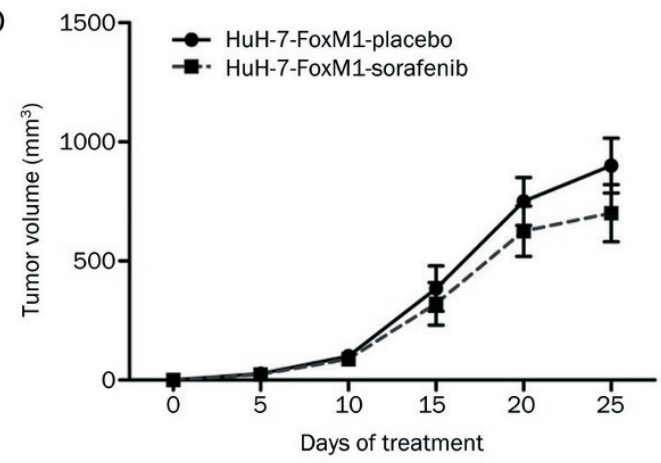

E

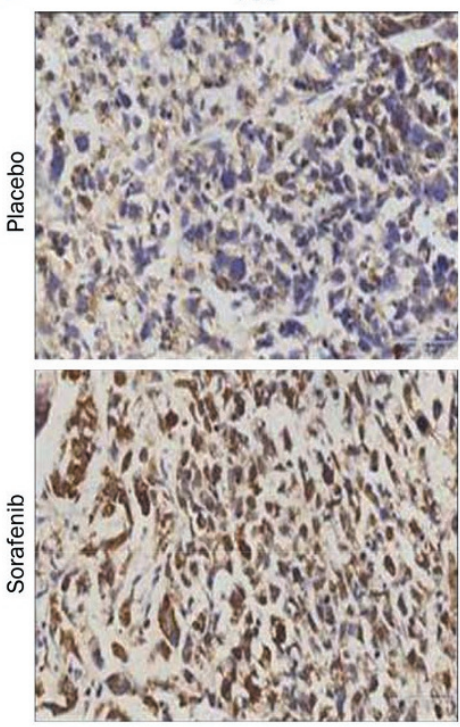

FoxM1
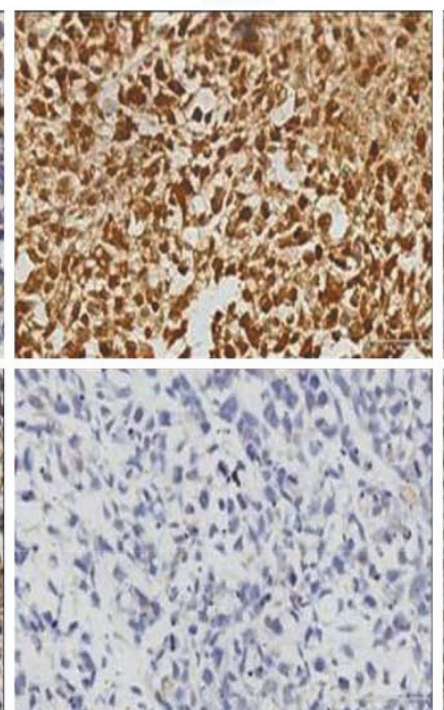

MMP-2
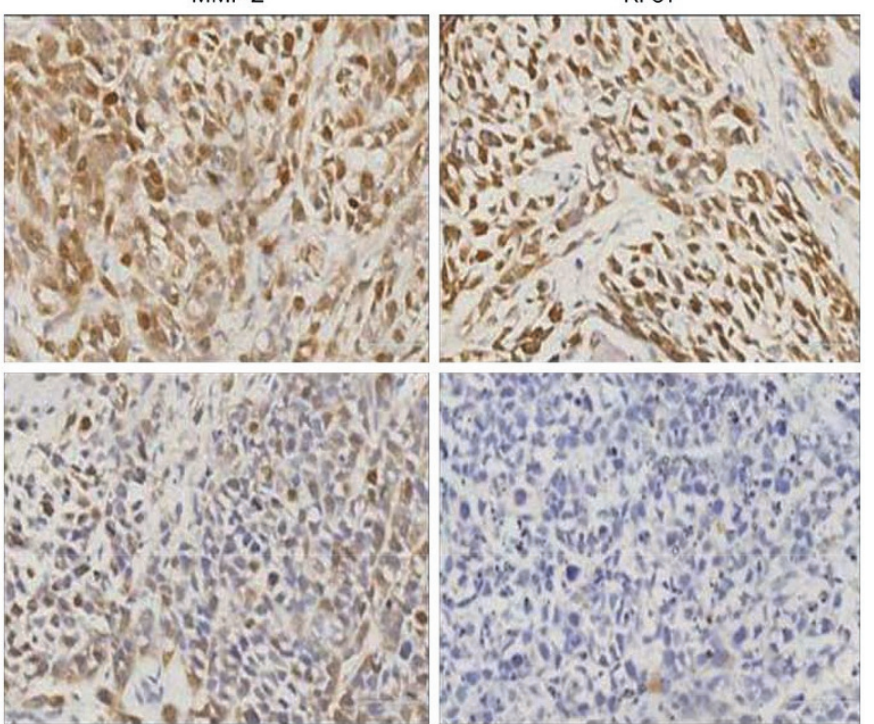

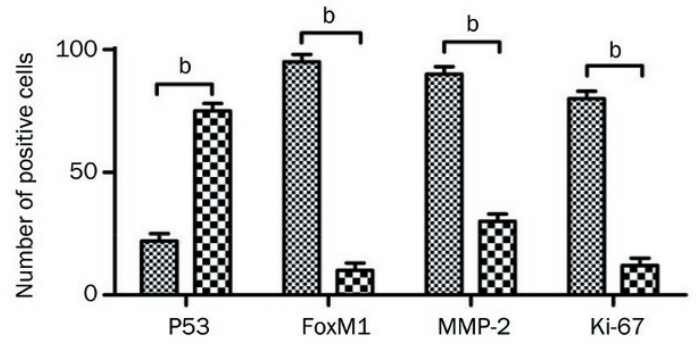

Figure 7. FoxM1 mediates the anti-tumor effect of sorafenib in a xenograft model. Effect of FoxM1 expression on tumor growth (A). Sorafenib inhibited tumor growth (B). Knocked-down FoxM1 expression in combination with sorafenib treatment strongly inhibited tumor size (C). The reduction in tumor size was not significant when FoxM1 expression was up-regulated in combination with sorafenib treatment (D). p53, FoxM1, MMP-2, and Ki-67 expression was determined via immunohistochemical analysis in tissues with and without sorafenib treatment. The number of positive cells was also quantified (E). Statistical significance was defined as ${ }^{\mathrm{b}} P<0.05$. 
inhibit FoxM1 ${ }^{[25]}$. One study reported that FoxM1 was inhibited by gefitinib ${ }^{[26]}$, an EGF receptor inhibitor. Another study showed that docetaxel significantly down-regulated FoxM1 $1^{[27]}$. Several proteasome inhibitors that have been clinically used have also been shown to down-regulate FoxM1 ${ }^{[28]}$. Furthermore, a p19 ${ }^{\mathrm{ARF}}$ peptide that significantly inhibits FoxM1 transcriptional activity efficiently diminished HCC proliferation and induced apoptosis of the HCC region in FoxM1 transgenic mice.

The multikinase inhibitor sorafenib has been approved by the FDA to treat $\mathrm{HCC}$, particularly unresectable $\mathrm{HCC}^{[3]}$. A previous study demonstrated that sorafenib significantly suppressed postsurgical intrahepatic recurrence and abdominal metastasis in an orthotopic HCC xenograft mouse model ${ }^{[29]}$. Sorafenib has frequently been shown to improve the surgical outcome of patients who have undergone HCC resection by effectively inhibiting tumor relapse and metastasis. Previous studies have reported that sorafenib blocks the Raf, PDGF, VEGF, FLT3, c-Kit, and MAPK signaling pathways ${ }^{[5]}$. Other reports have demonstrated that FoxM1 is the downstream target of MAPK signaling ${ }^{[30]}$, which can stimulate the nuclear translocation and transactivation activity of FoxM1. Theoretically, sorafenib can affect FoxM1 expression in HCC cells. However, the relationship between FoxM1 and sorafenib had not yet been demonstrated. FoxM1 plays an important role in cell cycle regulation ${ }^{[31]}$. Recent studies have shown that the prognosis and tumor recurrence may be associated with the expression of FoxM1, whose up-regulation can promote tumor proliferation by helping cells overcome the $\mathrm{G}_{1}-\mathrm{S}$ checkpoint ${ }^{[32]}$.

Our in vitro experiments suggest that sorafenib affects the expression of cell cycle regulatory factors, including E2F, CDK2, C-MYC, p53, and p27, all of which are FoxM1 targets. Previous studies have shown that E2F, CDK2, CDK4, and C-MYC promote cancer progression ${ }^{[33]}$. p27 and p53 have been shown to arrest the cell cycle and inhibit cancer cell proliferation $^{[34]}$. These results suggest that sorafenib inhibits the growth of HCC cells through cell cycle arrest and inhibits proliferation by suppressing FoxM1. To validate the relationship between sorafenib and FoxM1, we designed an in vivo experiment. We found that sorafenib showed dose-dependent antitumor activity in a murine xenograft model of human HCC that was accompanied by FoxM1 down-regulation.

The efficacy of sorafenib for treating HCC has been established in multicenter, phase III, double-blind, placebo-controlled trials. In some patients, tumor shrinkage appears to be less effective than expected ${ }^{[35]}$. In addition, a growing number of publications have reported complications associated with sorafenib treatment ${ }^{[36]}$. The occurrence of these adverse events may be prevented if the dosage of sorafenib required for effective treatment can be reduced. Therefore, it is necessary to select available targets that will improve the efficacy of chemotherapy so that these patients can benefit from sorafenib therapy. Recent studies have shown that FoxM1 contributes to chemotherapy resistance ${ }^{[20,21]}$. Another study demonstrated that FoxM1 mediates the gefitinib-induced proliferative arrest and determines sensitivity to gefitinib ${ }^{[26]}$. In our study, a high level of FoxM1 protein was correlated with sorafenib resistance in HCC cells. The HCC cells that expressed different levels of FoxM1 reacted differently when exposed to sorafenib. High FoxM1 expression was found in sorafenib-resistant cells, whereas low levels were observed in the comparatively sorafenib-sensitive cells.

These findings highlight an important molecular mechanism of action by which sorafenib enhances the therapeutic efficacy of cytotoxic agents. Therefore, FoxM1 has emerged as an attractive therapeutic target in chemotherapy resistance.

\section{Acknowledgements}

This study was supported by the National Natural Science Foundation of China (Grant № 30872482 and № 81072051) and the project of Innovative Research Team for Key Science and Technology in Xi'an Jiaotong University (No 2003KCJ-23).

\section{Author contribution}

Ji-chao WEI performed the majority of the experiments and wrote the manuscript; Fan-di MENG, Kai QU, Zhi-xin WANG, Ling-qiang ZHANG, Qing PANG, and Yue-lin ZHANG provided vital reagents and analytical tools; Qi-fei WU edited the manuscript; and Chang LIU designed the study and obtained financial support for the experiments.

\section{References}

1 Kim DY, Paik YH, Ahn SH, Youn YJ, Choi JW, Kim JK, et al. PIVKA-II is a useful tumor marker for recurrent hepatocellular carcinoma after surgical resection. Oncology 2007; 72: 52-7.

2 Scotto KW, Biedler JL, Melera PW. Amplification and expression of genes associated with multidrug resistance in mammalian cells. Science 1986; 232: 751-5.

3 Bruix J, Sherman M, American Association for the Study of Liver Diseases. Management of hepatocellular carcinoma: an update. Hepatology 2011; 53: 1020-24.

4 Lang L. FDA approves sorafenib for patients with inoperable Liver Diseases. cancer. Gastroenterology 2008; 134: 379.

5 Hahn O, Stadler W. Sorafenib. Curr Opin Oncol 2006; 18: 615-21.

6 Rini BI. Sorafenib. Expert Opin Pharmacother 2006; 7: 453-61.

7 Wilhelm S, Chien DS. BAY 43-9006: preclinical data. Curr Pharm Des 2002; 8: 2255-7.

8 Yu C, Bruzek LM, Meng XW, Gores GJ, Carter CA, Kaufmann SH, et al. The role of $\mathrm{Mcl}-1$ downregulation in the proapoptotic activity of the multikinase inhibitor BAY 43-9006. Oncogene 2005; 24: 6861-9.

9 Ho C, Wang C, Mattu S, Destefanis G, Ladu S, Delogu S, et al. AKT (v-akt murine thymoma viral oncogene homolog 1) and N-Ras (neuroblastoma ras viral oncogene homolog) coactivation in the mouse liver promotes rapid carcinogenesis by way of mTOR (mammalian target of rapamycin complex 1), FOXM1 (forkhead box M1)/SKP2, and c-Myc pathways. Hepatology 2012; 55: 833-45.

10 Wu QF, Liu C, Tai MH, Liu D, Lei L, Wang RT, et al. Knockdown of FoxM1 by siRNA interference decreases cell proliferation, induces cell cycle arrest and inhibits cell invasion in MHCC-97H cells in vitro. Acta Pharmacol Sin 2010; 31: 361-6.

11 Wang IC, Chen YJ, Hughes D, Petrovic V, Major ML, Park HJ, et al. Forkhead box $\mathrm{M} 1$ regulates the transcriptional network of genes essential for mitotic progression and genes encoding the SCF (Skp2Cks1) ubiquitin ligase. Mol Cell Biol 2005; 25: 10875-94.

12 Wang Z, Ahmad A, Li Y, Banerjee S, Kong D, Sarkar FH. Forkhead box 
M1 transcription factor: a novel target for cancer therapy. Cancer Treat Rev 2010; 36: 151-6.

13. Laoukili J, Stahl M, Medema RH. FoxM1: at the crossroads of ageing and cancer. Biochim Biophys Acta 2007; 1775: 92-102.

14 Ma RY, Tong TH, Leung WY, Yao KM. Raf/MEK/MAPK signaling stimulates the nuclear translocation and transactivating activity of FOXM1. In: Transcription Factors: Springer, 2010; 113-23.

15 Balli D, Zhang Y, Snyder J, Kalinichenko VV, Kalin TV. Endothelial cellspecific deletion of transcription factor FoxM1 increases urethaneinduced lung carcinogenesis. Cancer Res 2011; 71: 40-50.

16 Zhang Y, Zhang N, Dai B, Liu M, Sawaya R, Xie K, et al. FoxM1B transcriptionally regulates vascular endothelial growth factor expression and promotes the angiogenesis and growth of glioma cells. Cancer Res 2008; 68: 8733-42.

17 Wang Z, Banerjee S, Kong D, Li Y, Sarkar FH. Down-regulation of Forkhead Box M1 transcription factor leads to the inhibition of invasion and angiogenesis of pancreatic cancer cells. Cancer Res 2007; 67: 8293-300.

18 Tan Y, Raychaudhuri P, Costa RH. Chk2 mediates stabilization of the FoxM1 transcription factor to stimulate expression of DNA repair genes. Mol Cell Biol 2007; 27: 1007-16.

19 Myatt SS, Lam EW-F. The emerging roles of forkhead box (Fox) proteins in cancer. Nat Rev Cancer 2007; 7: 847-59.

20 Kwok JMM, Peck B, Monteiro LJ, Schwenen HDC, Millour J, Coombes $\mathrm{RC}$, et al. FOXM1 confers acquired cisplatin resistance in breast cancer cells. Mol Cancer Res 2010; 8: 24-34.

21 Carr JR, Park HJ, Wang Z, Kiefer MM, Raychaudhuri P. FoxM1 mediates resistance to herceptin and paclitaxel. Cancer Res 2010; 70: 5054-63.

22 Qu K, Xu X, Liu C, Wu Q, Wei J, Meng F, et al. Negative regulation of transcription factor FoxM1 by p53 enhances oxaloplatin-induced senescence in hepatocellular carcinoma. Cancer Lett 2012; 331: 105-14.

23 Sun H, Teng M, Liu J, Jin D, Wu J, Yan D, et al. FOXM1 expression predicts the prognosis in hepatocellular carcinoma patients after orthotopic liver transplantation combined with the Milan criteria. Cancer Lett 2011; 306: 214-22.

24 Limin X, Wenjie H, Dean T, Hongwu Z, Yongguo Z, Hao H, et al. Upregulated FoxM1 expression induced by hepatitis $B$ virus $X$ protein promotes tumor metastasis and indicates poor prognosis in hepatitis
B virus-related hepatocellular carcinoma. J Hepatol 2012; 57:60012.

25 Bhat UG, Halasi M, Gartel AL. Thiazole antibiotics target FoxM1 and induce apoptosis in human cancer cells. PLoS One 2009; 4: e5592.

26 McGovern UB, Francis RE, Peck B, Guest SK, Wang J, Myatt SS, et al. Gefitinib (Iressa) represses FOXM1 expression via FOXO3a in breast cancer. Mol Cancer Ther 2009; 8: 582-91.

27 Wang Z, Li Y, Ahmad A, Banerjee S, Azmi AS, Kong D, et al. Downregulation of Notch-1 is associated with Akt and FoxM1 in inducing cell growth inhibition and apoptosis in prostate cancer cells. J Cell Biochem 2011; 112: 78-88.

28 Crawford LJ, Walker B, Irvine AE. Proteasome inhibitors in cancer therapy. J Cell Commun Signal 2011; 5: 101-10.

29 Feng YX, Wang T, Deng YZ, Yang P, Li JJ, Guan DX, et al. Sorafenib suppresses postsurgical recurrence and metastasis of hepatocellular carcinoma in an orthotopic mouse model. Hepatology 2011; 53: 483-92.

30 de Olano N, Koo CY, Monteiro LJ, Pinto PH, Gomes AR, Aligue R, et al. The p38 MAPK-MK2 Axis regulates E2F1 and FOXM1 expression after epirubicin treatment. Mol Cancer Res 2012; 10: 1189-202.

31 Costa RH. FoxM1 dances with mitosis. Nat Cell Biol 2005; 7: 10810.

$32 \mathrm{Fu} \mathrm{Z,} \mathrm{Malureanu} \mathrm{L,} \mathrm{Huang} \mathrm{J,} \mathrm{Wang} \mathrm{W,} \mathrm{Li} \mathrm{H,} \mathrm{van} \mathrm{Deursen} \mathrm{JM,} \mathrm{et} \mathrm{al.}$ Plk1-dependent phosphorylation of FoxM1 regulates a transcriptional programme required for mitotic progression. Nat Cell Biol 2008; 10: 1076-82.

33 Beier R, Bürgin A, Kiermaier A, Fero M, Karsunky H, Saffrich R, et al. Induction of cyclin E-cdk2 kinase activity, E2F-dependent transcription and cell growth by Myc are genetically separable events. EMBO J 2000; 19: 5813-23.

34 Leung KC, Hsin MKY, Chan JSY, Yip JHY, Li M, Leung B, et al. Inhibition of thromboxane synthase induces lung cancer cell death via increasing the nuclear p27. Exper Cell Res 2009; 315: 2974-81.

35 Cheng AL, Kang YK, Chen Z, Tsao CJ, Qin S, Kim JS, et al. Efficacy and safety of sorafenib in patients in the Asia-Pacific region with advanced hepatocellular carcinoma: a phase III randomised, double-blind, placebo-controlled trial. Lancet Oncol 2009; 10: 25-34.

36 Kong HH, Sibaud V, Chanco Turner ML, Fojo T, Hornyak TJ, et al. Sorafenib-induced eruptive melanocytic lesions. Arch Dermatol 2008; 144: 820-2. 